\title{
Regulation of synaptic architecture and synaptic vesicle pools by Nervous wreck at Drosophila Type 1b glutamatergic synapses
}

\author{
Joon Haeng Hur ${ }^{1,2,4}$, Sang-Hee Lee ${ }^{3,4}$, A-Young Kim ${ }^{1,2,4}$ and Young Ho Koh ${ }^{1,2}$
}

Nervous wreck (Nwk), a protein that is present at Type 1 glutamatergic synapses that contains an SH3 domain and an $\mathrm{FCH}$ motif, is a Drosophila homolog of the human srGAP3/MEGAP protein, which is associated with mental retardation. Confocal microscopy revealed that circles in Nwk reticulum enclosed T-shaped active zones (T-AZs) and partially colocalized with synaptic vesicle (SV) markers and both exocytosis and endocytosis components. Results from an electron microscopic (EM) analysis showed that Nwk proteins localized at synaptic edges and in SV pools. Both the synaptic areas and the number of SVs in the readily releasable (RRPs) and reserve (RPs) SV pools in $n w^{2}{ }^{2}$ were significantly reduced. Synergistic, morphological phenotypes observed from eag ${ }^{1} ; n w^{2}{ }^{2}$ neuromuscular junctions suggested that Nwk may regulate synaptic plasticity differently from activitydependent Hebbian plasticity. Although the synaptic areas in eag $^{1} ;$;nwk ${ }^{2}$ boutons were not significantly different from those of $n w k^{2}$, the number of SVs in the RRPs was similar to those of Canton-S. In addition, three-dimensional, high-voltage EM tomographic analysis demonstrated that significantly fewer enlarged SVs were present in $n w^{2}{ }^{2}$ RRPs. Furthermore, Nwk formed protein complexes with Drosophila Synapsin and Synaptotagmin 1 (DSypt1). Taken together, these findings suggest that Nwk is able to maintain synaptic architecture and both SV size and distribution at T-AZs by interacting with Synapsin and DSypt1. Experimental \& Molecular Medicine (2018) 50, e462; doi:10.1038/emm.2017.303; published online 23 March 2018

\section{INTRODUCTION}

Normal development, maintenance and plasticity of neuronal circuits and synaptic architectures are critical for proper brain functions in humans and other animals. ${ }^{1,2}$ Defects in one or more genes involved with neuronal wirings or with synapse formation, development, differentiation, plasticity or transmission have been identified as the causes of many neurological disorders, including mental retardations, ${ }^{3}$ Fragile X-syndrome, ${ }^{4}$ Rett syndrome, ${ }^{5}$ autism, ${ }^{6}$ Angelman syndrome ${ }^{7}$ and mild learning disability. ${ }^{8}$ The causative mutations may induce abnormal development of neuronal connections or synaptic architectures by perturbing one or more molecular or cellular mechanisms underlying homeostatic regulatory mechanisms, Hebbian plasticity or both. ${ }^{2,8}$ Hebbian plasticity is the idea that strengthening or weakening synaptic efficacy in neuronal circuits is mainly determined by correlated presynaptic and postsynaptic activities. ${ }^{9}$ Increased neuronal excitabilities can strengthen neuronal circuits by increasing the sizes of synapses, and decreased neuronal activities can eliminate synaptic connections. Activity-dependent Hebbian plasticity may explain how long-term potentiation or depression is accomplished without extensive genetic instruction. Nevertheless, without a homeostatic regulatory mechanism keeping neuronal circuits from runaway excitation or quiescence, Hebbian plasticity may not be sufficient to explain how synaptic architectures and neuronal circuits in the brains maintain excitability within physiological limits during learning and development. ${ }^{9-11}$ Homeostatic regulatory mechanisms are considered to have important roles in maintaining some consistency in neuronal properties despite the changes induced by activity-dependent Hebbian plasticity by altering preneuronal and/or postneuronal properties, such as synaptic vesicle (SV) release probability, synapse sizes, neurotransmitter receptor localization or retrograde signaling. ${ }^{10,12}$

Because the basic functional unit in the brain is a single synapse, ${ }^{13,14}$ in order to understand the molecular and cellular mechanisms underlying synaptic plasticity and the molecular and cellular etiologies underlying neuronal disorders,

\footnotetext{
${ }^{1}$ ILSONG Institute of Life Science, Hallym University, Anyang, Republic of Korea; ${ }^{2}$ Department of Bio-Medical Gerontology, Hallym University Graduate School, Chuncheon, Republic of Korea and ${ }^{3}$ BioMedical Research Center, Korea Advanced Institute of Science and Technology, Daejeon, Republic of Korea 4JH Hur, S-H Lee and A-Y Kim contributed equally to this work. Correspondence: Professor YH Koh, ILSONG Institute of Life Science, Hallym University, Anyang, Gyeonggi-do 431-060, Korea. E-mail: Kohyh@hallym.ac.kr Received 25 June 2017; revised 29 August 2017; accepted 29 September 2017
} 
identifying the molecular components localizes at synapses and investigating their roles in vivo are among the major foci in modern molecular neuro-genetics. The basic molecular components and neuronal signaling pathways in excitatory synapses in the human brains have been shown to be faithfully conserved at Type 1 glutamatergic synapses in Drosophila larval neuromuscular junctions (NMJ). ${ }^{15-18}$ Therefore, the molecular and cellular mechanisms underlying synaptic plasticity have been intensively investigated in Type 1 glutamatergic synapses in Drosophila larval NMJs, the best-characterized synapses. A plethora of studies in Drosophila larval NMJs have shown that homeostatic regulatory mechanisms and Hebbian plasticity are present and actively participate in regulating the development, differentiation and plasticity of neuronal circuits and synapse architectures. Genetic alterations in neuronal activity have resulted in either increased or decreased synapse sizes in larval NMJs, ${ }^{19-21}$ accompanied by alterations in the localization and composition of synaptic molecular components that form postsynaptic or presynaptic terminal macromolecular complexes. ${ }^{22,23}$ Synaptic macromolecular complexes harbor synaptic scaffolding proteins, cell adhesion molecules, ion channels, various protein kinases and neurotransmitter receptors. ${ }^{24}$ In addition, SVs are linked to active zones (including exocytosis and endocytosis machineries) by proteins regulating the trafficking of SVs from the reserve pool (RP) to readily releasable pool (RRP). ${ }^{25,26}$ The in vivo functions of evolutionary conserved molecular components present at synapses were revealed by studies using available mutant alleles in Drosophila. Discs large (DLG), the Drosophila homolog of membrane-associated guanylated kinase (MAGUK) family proteins, are critical for the proper targeting and localization of Shaker type $\mathrm{K}^{+}$channels, ${ }^{27} \mathrm{Ca}^{2+} /$ calmodulin-dependent kinase $\mathrm{II}^{28}$ and the Drosophila cell adhesion molecule, Fasciclin II (FasII) ${ }^{29}$ at synapses. Mutations in various $\mathrm{K}^{+}$channels are resulted in increased sizes of synapses at larval NMJs, a typical example of Hebbian plasticity. In addition, hyperpolarization of muscle resting potentials via transgenic expression of inward rectifier $\mathrm{K}^{+}$channel Kir2.1 induced increased presynaptic release of neurotransmitters to produce normal levels of depolarization, a typical example of homeostatic regulatory mechanisms. ${ }^{30}$ Furthermore, CaMKII and cAMP-dependent protein kinase have been shown to be involved with homeostatic regulatory mechanisms via regulating retrograde signaling that alters the physiological properties at the presynaptic terminals.

Nervous wreck (Nwk), a SRC homology 3 (SH3) domain and Fes/Cip4 homology-Bin/Amphiphysin/Rvs167 (F-Bar) domain containing protein present at Type 1 glutamatergic synapses in reticular structures, is a Drosophila homolog of human SLIT-ROBO Rho GTPase activating protein 3 (srGAP3)/mental disorder associated GTPase-acting protein (MEGAP), which has been linked to severe mental retardation. ${ }^{3} n w k$ was identified as a negative regulator of structural and functional synaptic plasticity. ${ }^{31}$ Key morphological phenotypes in NMJs in $n w k$ loss-of-function mutants, such as increased numbers of synaptic bouton, were similar to those observed from mutations inducing increased neural activity, a classical example of Hebbian plasticity. However, the existence of other phenotypes, such as smaller synaptic boutons and synapses that were different from NMJs in hyperexcitability mutants suggested that $n w k$ may not participate in canonical activity-dependent Hebbian plasticity. In this study, we provide genetic, anatomical and biochemical evidence supporting our hypothesis that 'Nwk regulates synaptic plasticity by regulating synapse architecture and maintaining SV sizes and pools'.

\section{MATERIALS AND METHODS}

\section{Fly genetics}

Flies were raised on standard Drosophila media at $25 \pm 1{ }^{\circ} \mathrm{C}$ with $60 \%$ relative humidity. Previously characterized Canton-S (CS), nervous wreck $^{2}\left(n w k^{2}\right), D f(3 L) R d l 2,{ }^{31}$ Fasciclin $I^{e 76}\left(\right.$ FasII $\left.^{e 76}\right),{ }^{29}$ ether a go-go ${ }^{1}$ $\left(e a g^{l}\right)$ and UAS-neuronal-synaptobrevin-green fluorescence protein (n-Syb-GFP), UAS-Actin-GFP (Bloomington Drosophila Stock Center, Indiana University, Bloomington, IN, USA) were used in this study. $e a g^{1} ; ; n w k^{2}$ and fasII $I^{e 76} ; n w k^{2}$ double mutants were generated by recombining the first and third chromosomes.

\section{Immunohistochemistry}

Wandering-stage third-instar larvae were dissected in ice-cold $\mathrm{Ca}^{2+}$ free saline (128 mM NaCl, $2 \mathrm{~mm} \mathrm{KCl,} 4 \mathrm{~mm} \mathrm{MgCl} 2,35.5 \mathrm{~mm}$ sucrose, 5 mм HEPES, 1 mм EGTA, pH 7.2; Sigma-Aldrich, St Louis, MO, USA) and fixed with $4 \%$ paraformaldehyde in $0.1 \mathrm{~m}$ phosphate buffer $(\mathrm{PB}, \mathrm{pH} 7.2)$ for $1 \mathrm{~h}$ at room temperature. ${ }^{32}$ Goat anti-horseradish peroxidase (HRP)-fluorescein isothiocyanate, ${ }^{33}$ rabbit anti-Nwk, ${ }^{31}$ mouse monoclonal antibody (Mab)-Bruchpilot (Brp, Developmental Studies Hybridoma Bank (DSHB), University of Iowa, Iowa City, IA, USA), Mab-Fasciclin II (FasII, DSHB), Mab-Synapsin (DSHB), Mab-Drosophila cysteine string protein (Dcsp; DSHB), rabbit-antiLike-clathrin adaptor protein 180 (Lap), ${ }^{34}$ Mab-Futsch (DSHB), Mab-Neuroglian (DSHB), Mab-Highwire (Hiw, DSHB), Mab-Drosophila glutamate receptor IIA (DGluRIIA, DSHB), Mab-Discs larges 1 (DLG1, DSHB), Mab-Drosophila synaptotagmin 1 (DSypt1, DSHB), rabbit anti-DAP $160^{35}$ and rabbit anti-Drosophila P-21 activated kinase (Dpak) were used. Donkey anti-mouse Alexa-488 and anti-rabbitAlexa 568 (ThermoFisher Scientific, Waltham, MA, USA) were used as secondary antibodies. The numbers of synaptic boutons on muscles 6 and 7 in the abdominal segment 2 were counted under epifluorescence microscopy. The lengths of synaptic branches and sizes of synaptic boutons were measured using measure functions in $\mathrm{NIH}$ Image J (Bethesda, MD, USA). ${ }^{36}$ A Zeiss LSM700 confocal microscope (Zeiss, Oberkorchen, Germany) was used to acquire images of samples. Images were processed with the Zen (Zeiss) and Adobe Photoshop (Adobe, San Jose, CA, USA) software.

The relative intensities of anti-FasII immunoreactivity were calculated from single-slice confocal images of synaptic boutons on muscles 6 and 7 in the abdominal segment 2 processed with the antiFasII antibody 1D4 under similar conditions. Briefly, the longitudinal axis of the synaptic boutons were decided and lines at an angle of approximately $0^{\circ}, 90^{\circ}, 180^{\circ}$ and $270^{\circ}$ from that axis were traced from the center of the synaptic bouton to the outer limit occupied by FasII immunoreactivity. Then the maximum intensity along lines (on a linear scale of 0-255) was determined using the Plot Profile function of Scion Image analysis program (version 1.62). To quantify FasII immunoreactivity at synaptic boutons, 57 synaptic boutons in eag ${ }^{1}$, 
83 in CS, 70 in $n w k^{2}, 79$ in $n w k^{2} / D f$ or 60 in eag $; ; n w k^{2}$ from 4 independent NMJs processed simultaneously were used for quantification. One-way analysis of variance with Tukey's post hoc test was performed using Excel (Microsoft, Redmond, WA, USA) to analyze the data.

\section{Immuno-electron microscopy}

Third-instar wandering-tage control and mutant larvae were dissected in ice-cold $\mathrm{Ca}^{2+}$-free saline and then fixed with $0.005 \%$ glutaraldehyde and $4 \%$ paraformaldehyde in $0.1 \mathrm{M} \mathrm{PB}$ for $1 \mathrm{~h}$. Body wall muscle preparations were marked and processed in the same well. After washing three times with $0.1 \mathrm{M}$ PB with $0.2 \%$ Triton-X 100 (PBT), samples were incubated with rabbit anti-Nwk antibodies (diluted 1:2,000) overnight at $4{ }^{\circ} \mathrm{C}$. After being blocked with $4 \%$ bovine serum albumin and $4 \%$ normal goat serum in $0.1 \mathrm{M} \mathrm{PB}$, tissues were incubated with anti-rabbit-IgG conjugated to $1 \mathrm{~nm}$ gold (1:200 diluted; Nanoprobes.com, Yaphank, NY, USA). After washing three times with PBT, silver enhancement of nano-gold particles was performed according to the manufacturer's recommendation (Nanoprobes) for $10 \mathrm{~min}$. After washing with $\mathrm{H}_{2} \mathrm{O}$ for $5 \mathrm{~min}$, samples were incubated with gold enhancement solution for $5 \mathrm{~min}$. Gold enhancement was used to prevent silver deposits from etching. Silver- and gold-enhanced tissues were fixed with $1 \%$ glutaraldehyde and $4 \%$ paraformaldehyde in $0.1 \mathrm{M} \mathrm{PB}$ overnight. Tissues were washed three times with $\mathrm{dH}_{2} \mathrm{O}$, fixed with $1 \% \mathrm{OsO}_{4}$ in $0.1 \mathrm{M} \mathrm{PB}$ for $1 \mathrm{~h}$ and then dehydrated in serial EtOH and 100\% Propylene-oxide (Ted Pella Inc., Redding, CA, USA). Tissues were embedded with Spurr's resin (Ted Pella Inc.) and polymerized at $60^{\circ} \mathrm{C}$ overnight. The membrane-coated one-slot grids were used to collect serial thin sections $(100 \mathrm{~nm})$ and observed under a Jeol 1011 (Jeol Inc., Tokyo, Japan). Only Type 1b synaptic boutons on muscle 6 and 7 in the abdominal segment 2 were used for examination.

\section{Western blot analysis}

Ten wandering-stage third-instar larvae were dissected in ice-cold $\mathrm{Ca}^{2+}$ free saline and homogenized in ice-cold radio-immunoprecipitation assay (RIPA) buffer ( $150 \mathrm{~mm} \mathrm{NaCl}, 1 \%$ IGEPAL CA-630, $0.5 \%$ Na-oxycholate, $0.1 \%$ sodium dodecyl sulfate (SDS), $50 \mathrm{~mm}$ Tris, $\mathrm{pH}$ 8.0; Sigma-Aldrich) with protease inhibitors (Halt protease inhibitor cocktail, ThermoFisher Scientific, Rockford, IL, USA). After being centrifuged at $14000 \mathrm{~g}$ for $20 \mathrm{~min}$ at $4{ }^{\circ} \mathrm{C}$ to remove cellular debris, the amount of protein in each supernatant was determined using the BCA Protein Assay Kit (ThermoFisher Scientific, Waltham, MA, USA). A total of $20.0 \mu \mathrm{g}$ of protein from each sample was separated on a $10 \%$ tris-glycine polyacrylamide SDS gel and then transferred to a nitrocellulose membrane (GE Healthcare Bio-Sciences AB, Uppsala, Sweden). The membranes were probed with rabbit antiNwk antibodies. Mouse anti- $\alpha$-tubulin antibody was used as a loading control.

\section{Coimmunoprecipitation analysis}

Twenty wandering-stage third-instar larvae were dissected in ice-cold $\mathrm{Ca}^{2+}$ free saline and homogenized in ice-cold RIPA buffer with protease inhibitors (Halt protease inhibitor cocktail, ThermoFisher Scientific, Rockford, IL, USA). Supernatants were collected by spinning down at $14000 \mathrm{~g}$ for $15 \mathrm{~min}$ at $4{ }^{\circ} \mathrm{C}$. Protein $\mathrm{A}+\mathrm{G}$ agarose beads (SantaCruz Biotech., San Jose, CA, USA) were blocked with RIPA buffer with $1 \%$ bovine serum albumin for $2 \mathrm{~h}$ with rolling at $4{ }^{\circ} \mathrm{C}$. After being washed three times with RIPA buffer, protein $\mathrm{A}+\mathrm{G}$ agarose beads were mixed with the supernatants, which contained $20 \mu$ of
Mab-Synapsin and were rocked overnight at $4{ }^{\circ} \mathrm{C}$. Protein $\mathrm{A}+\mathrm{G}$ agarose beads were harvested by centrifugation at $14000 \mathrm{~g}$ for 10 min at $4{ }^{\circ} \mathrm{C}$, washing three times with RIPA buffer and then boiling with $1 \times$ SDS sample buffer. Proteins were separated by $10 \%$ SDS-polyacrylamide gel electrophoresis and immediately transferred onto nitrocellulose membranes. Membranes were probed with rabbit anti-Nwk, Mab-DSypt1 and Mab-Synapsin.

\section{Transmission electron microscopy}

The protocols for transmission EM and morphometric analysis of synaptic boutons have been previously published. ${ }^{33}$ For this experiment, serial thin sections $(100 \mathrm{~nm})$ were collected from only Type $1 \mathrm{~b}$ glutamatergic synaptic boutons on muscle 6 and 7 (abdominal segment 2). The thickness of thin sections was confirmed by examining wrinkles of thin sections at $15000 \times$ with a Jeol 1011 (Jeol Inc.).

\section{Three-dimensional tomography of synapses with high- voltage electron microscope}

Thick sections $(1 \mu \mathrm{m})$ were collected from CS and $n w k^{2}$ mutant synaptic boutons. Then $20 \mathrm{~nm}$ colloidal gold was applied on the surface of thick sections to create a list of fiducial points and used as a standard for determining sizes of SVs and T-shaped active zones (T-AZs). The specimens were tilted from $-60^{\circ}$ to $+60^{\circ}$ and imaged at $1^{\circ}$ steps (121 images per view) using a high-voltage electron microscope (HVEM; Jeol) operated at 1 MV. Tilted images were aligned using the IMOD software ${ }^{37}$ to visualize three-dimensional (3D) structures of synapses and active zones. The presynaptic and postsynaptic membranes, SVs and T-AZs were visualized. The sizes and distances of SVs from active zones were analyzed. One-way T-tests were performed by Excel (Microsoft) for statistical analyses.

\section{RESULTS}

Nwk reticulum localizes at the peri-synaptic area and SVs The precise localization patterns of Nwk at Type I glutamatergic boutons in Drosophila larval NMJs were examined by performing anti-Nwk immunocytochemistry. The specificity of anti-Nwk in immunocytochemistry was confirmed by using $n w k^{2}$ null mutants (Supplementary Figure 1). Nwk signals were observed mainly at the presynaptic terminals of Type I boutons and did not overlap with DLG signals in wild-type CS NMJs (Supplementary Figure 1). DLG has been shown to be present mainly at the postsynaptic terminals of Type I boutons that have elaborated and convoluted membrane structures, named subsynaptic reticulum (SSR). ${ }^{28,29}$ However, the outer boundaries of Nwk immuno-reactivity overlapped with anti-HRP signals, indicating neuronal membrane structures (Supplementary Figure 1$)^{38}$ and suggesting that Nwk is distributed within the presynaptic terminals of Type I boutons at the confocal microscopic level. No detectable Nwk signals were observed from NMJs or muscles of $n w k^{2}$ animals (Supplementary Figure 1C2), even though anti-DLG and anti-HRP signals appeared to be normal in $n w k^{2}$ NMJs (Supplementary Figure 1C1).

The localization patterns of Nwk at synapses in Type $1 \mathrm{~b}$ boutons in CS were further revealed by serial double-labeling confocal microscopy employing antibodies targeting Nwk and other synaptic or cytoskeletal components (Figure 1). Nwk 
signals, indicated in red in Figure 1, were distributed at the presynaptic terminals in reticular structures that were formed of several interlinked circles and termed Nwk reticulum. Nwk reticulum at the presynaptic terminal was observed from the surface (Figure 1b) through the center of synaptic boutons (Figures 1a and c-n). Narrow passages clear of Nwk signals are present in the middle of boutons indicated by gray arrow heads (Figure 1a and c-e). A single-slice confocal image taken from
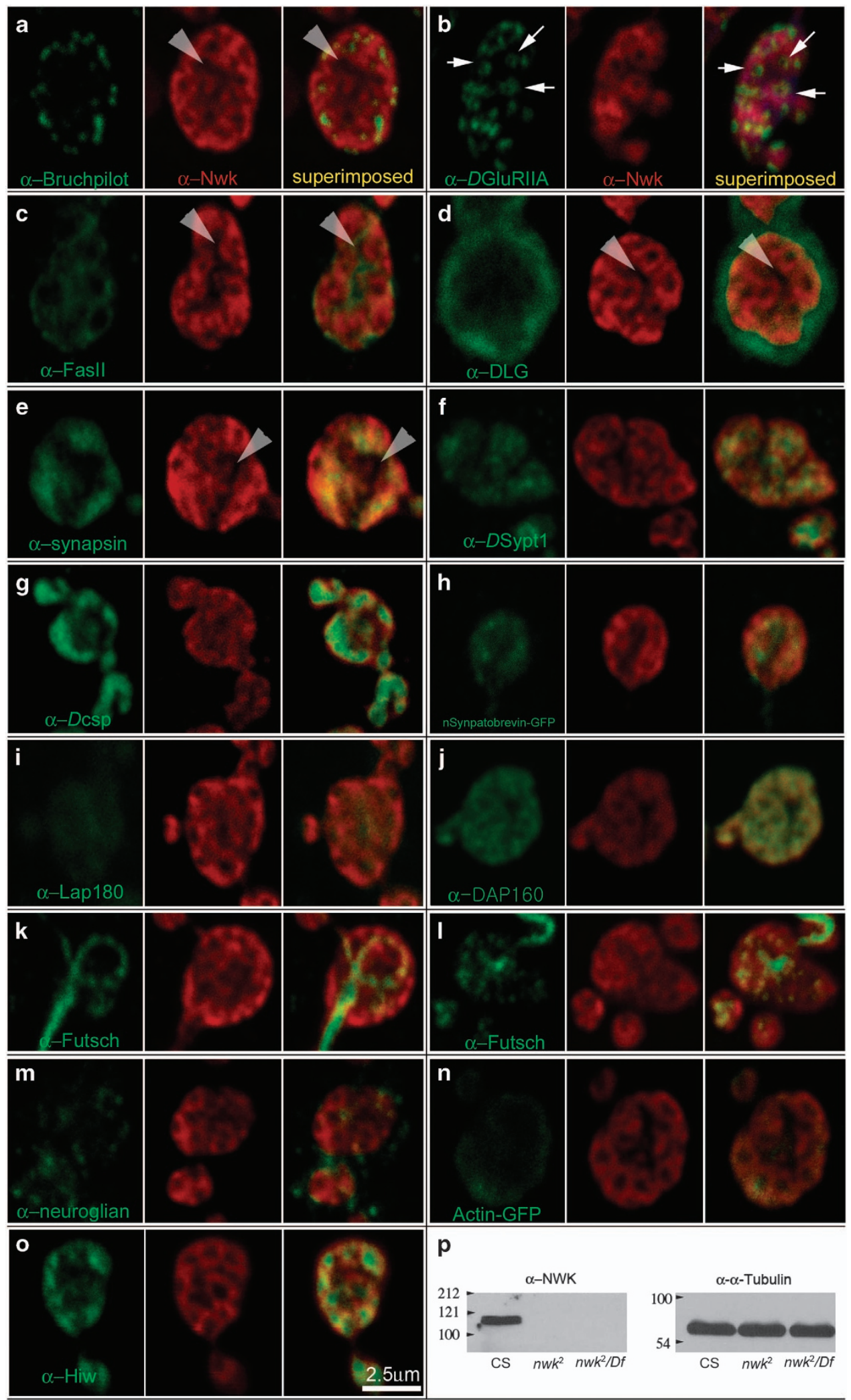
the midline of a bouton double-labeled for anti-Nwk and the T-AZ marker, Mab-Brp, ${ }^{39,40}$ indicated that Nwk circles were present at the peri-active zones (Figure 1a). Double-labeling with a postsynaptic density maker (Mab-DGluRIIA) ${ }^{17}$ showed that Nwk circles directly faced the edges of postsynaptic densities. There was a hole at the center of DGluRIIA signals corresponding to T-AZs (Supplementary Figure 2A) indicated by a white arrow (Figure $1 \mathrm{~b}$ ). When another postsynaptic density marker, anti-Drosophila p-21 activated protein kinase (Dpak, red), was used for double-labeling with Mab-Brp (green), T-AZs were localized at the center of postsynaptic density (Supplementary Figure 2C). FasII, a Drosophila homolog of cell adhesion molecule has been shown to be localized on both the presynaptic and postsynaptic membranes in Type I glutamatergic NMJs. ${ }^{22,29}$ Anti-Nwk and Mab-FasII doublelabeling experiments showed that Nwk reticulum and FasII signals are not overlapped (Figure 1c). FasII signals were observed surrounding circles in the Nwk reticulum but were not present on the inner sides of Nwk circles. In addition, FasII was present at the narrow passages in the middle of synaptic boutons that were clear of Nwk signals pointed by gray arrow heads. In addition, FasII signals did not colocalize with MabBrp signals (Supplementary Figure 2B). These distribution patterns suggested that Nwk circles are closer to T-AZs than FasII signals at the presynaptic membranes and may regulate synapse stability. Double-labeling with a postsynaptic terminal maker (anti-DLG) showed that the Nwk reticulum was restricted to within the presynaptic terminals of Type $\mathrm{Ib}$ boutons (Figure 1d).

Distribution patterns of Nwk reticulum at the presynaptic terminals were further analyzed by using SV markers, such as Mab-Synapsin (Figure 1e), anti-DSypt1 (Figure 1f) or Mab-Dcsp (Figure 1g); molecular components of exocytosis compartments, such as n-syb-GFP (Figure 1h), and endocytosis markers, such as anti-LAP180 (Figure 1i) and anti-Dap 160 (Figure 1j). Nwk reticulum was partially colocalized with SV markers
(Figures 1e-g), n-syb-GFP signals (Figure 1h) and LAP-180 signals (Figure 1i), as indicated by the yellow colors clearly visible in the merged images. Interestingly, anti-Dap160 and anti-Nwk signals partially colocalized at Type Ib boutons (Figure 1j). Previous studies in dap160 loss-of-function mutant showed that Nwk localizations at boutons was impaired, even though Dap160 localization at $n w k^{2}$ boutons was not clearly perturbed. ${ }^{41}$

To understand how Nwk reticulum is linked to the cytoskeleton in the presynaptic terminals, the microtubule marker Mab-Futsch (Figures $1 \mathrm{k}$ and 1) and Actin-GFP (Figure $1 \mathrm{~m}$ ) were used to reveal cytoskeleton networks in the boutons. Presynaptic Futsch-microtubule structures traversed the synaptic boutons that were not undergoing division or sprouting (Figure 1k). However, synaptic boutons undergoing division or sprouting were usually present at the end of synaptic branches and did not have Futsch-microtubule cytoskeleton loops. Instead, Futsch-microtubule signals were diffuse and present at the center of Nwk circles, indicating that the destabilization of Futsch-microtubule loop structures is a critical process for dividing or sprouting synaptic boutons (Figure 11). ${ }^{42}$ These results suggested that Nwk reticulum is not directly linked to microtubules. However, Nwk reticulum at the bouton midline were partially colocalized with Actin-GFP signals, reinforcing previous reports that interactions between Nwk and the Actin cytoskeleton are important for structural and functional synaptic plasticity at the Drosophila larval NMJs. ${ }^{31}$ Neuroglian, the Drosophila L1 homolog shown to be involved with axon pathfinding, was present on both the presynaptic and postsynaptic terminals but was not colocalized with Nwk in Type 1 b boutons (Figure $1 \mathrm{~m}$ ). In addition, Nwk reticulum partially colocalized with Hiw, shown to be related with FasII-independent synaptic plasticity (Figure 1o). Western blots from larval body wall muscle extracts of CS, $n w k^{2}$ and $n w k^{2} / D f$ were probed sequentially with anti-Nwk or Mab- $\alpha$ Tubulin (12G10) (Figure 1p). Two strong NWK bands of approximately $110 \mathrm{Kda}$ that were clearly seen from CS body

Figure 1 Nwk reticulum at synaptic boutons in Drosophila larval Type $1 \mathrm{~b}$ boutons in neuromuscular junctions (NMJs). To reveal the precise localization patterns of Nwk at the presynaptic terminals of Type 1 glutamatergic NMJs, Nwk antibodies (red) were counter-stained with antibodies targeting other identified synaptic molecular components or cytoskeleton components (green). All images are single-slice confocal images taken from a midline (a, c-n) or a surface of synaptic boutons (b). (a) T-AZs labeled with Mab-Brp (green) were localized at the center of circles in Nwk reticulum (red) clearly seen from a merged image. (b) A postsynaptic density marker, Mab-DGluRIIA (green) and anti-Nwk (red) indicated that PSDs localized at the center of circles in Nwk reticulum clearly seen in the merged image. (c) Mab-Fasll (green) signals in a midline of synaptic bouton surrounded the Nwk reticulum (red) and localized at the Nwk-negative narrow passage indicated by white arrow heads. (d) Double labeling with Mab-DLG, a postsynaptic terminal structure marker (green) and anti-Nwk (red) revealed that Nwk reticulum is present at the presynaptic terminals. (e, $\mathbf{g}, \mathbf{h}$ ) Antibodies of SV proteins (green), such as Mab-synapsin(e), anti-DSypt1 (g) or Mab-Dcsp2 (h) were present at the center of circles in Nwk reticulum (red) but not in the Nwk-negative narrow passages indicated by white arrow head. (f) When n-Syb-GFP was expressed using the pan-neuronal C155-Gal4 driver, n-Syb-GFP (green) was partially colocalized with Nwk reticulum (Red). (i) Anti-LAP180 (green) was partially colocalized with Nwk reticulum. (j) Mab-Hiw partially colocalized with the Nwk reticulum. Particularly strong Hiw signals were present at the center of Nwk circles (red). (k, I) Mab-Futsch revealed microtubules in boutons. In the stable synaptic boutons, microtubule-Futsch loops traversing the middle of synaptic boutons were clearly visible (k). Synaptic boutons undergoing sprouting or dividing did not have microtubule-Futsch loop structures (I). Instead, microtubule-Futsch signals were present at the centers of circles in NWK reticulum. (m) Drosophila L1 homolog, Neuroglian (Mab-BP104, green), did not colocalize with Nwk reticulum at the synaptic boutons. (n) When Actin-GFP was expressed in the neurons using C155Gal4, Actin-GFP proteins (green) was partially colocalized with Nwk reticulum. (o) Nwk reticulum partially colocalized with Hiw. (p) A western blot from larval body wall muscle extracts of CS, $n w k^{2}$ and $n w k^{2} / D f$ was probed sequentially with anti-Nwk or Mab- $\alpha$-Tubulin (12G10). There was no Nwk expression in $n w k^{2}$ and $n w k^{2} / D f$. 


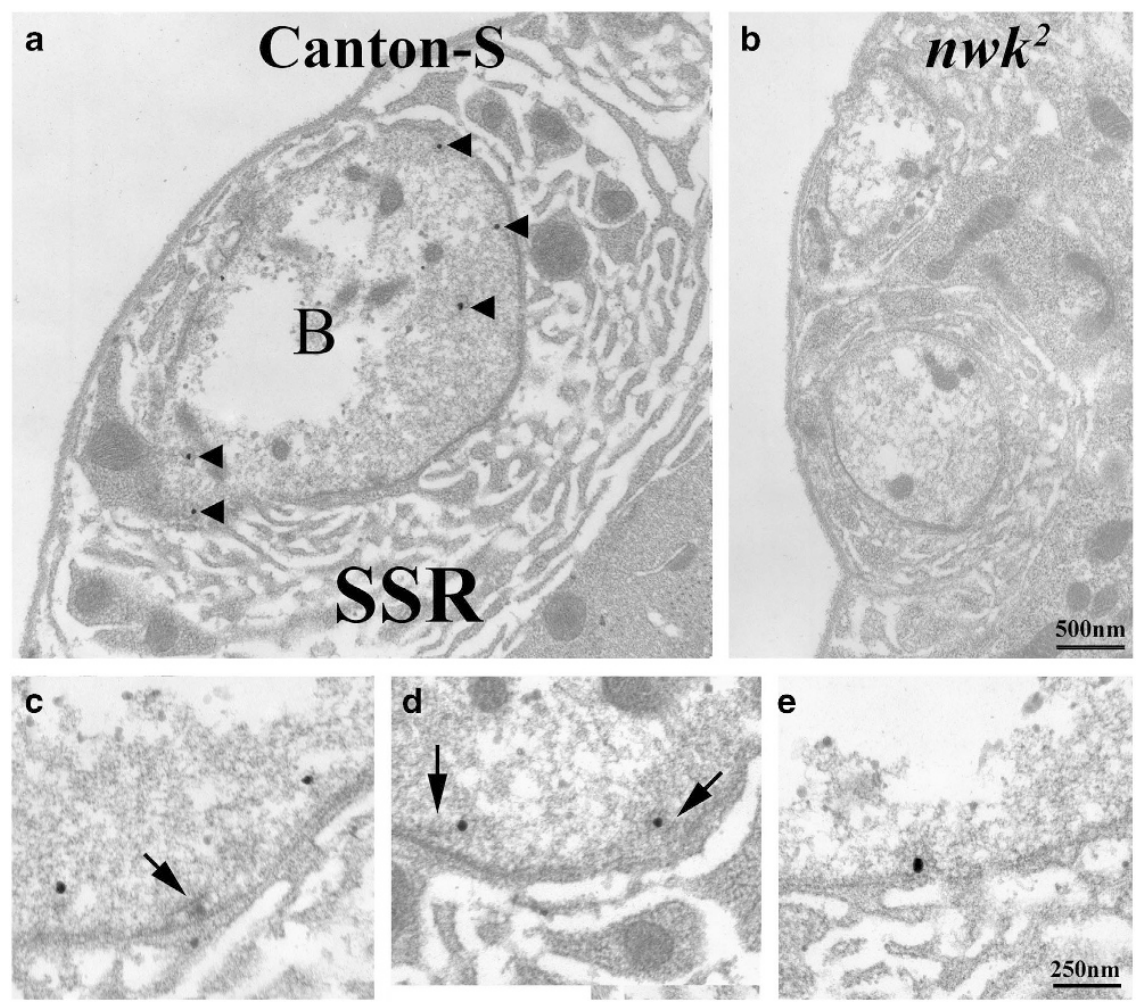

Figure 2 Nwk is localized at the edges of the synapses and in SV pools. (a) Anti-Nwk-positive silver- and gold-enhanced particles indicated by black arrow heads were localized at the edge of synapses or within SV pools in CS synaptic boutons. (b) However, no anti-Nwk-positive particles were present in $n w k^{2}$ synaptic boutons. (c-e) Nwk particles were localized within reserve SV pools (c), readily releasable SV pools (d) or the edge of a synapse (e). Black arrows indicate T-AZ at synapses. B, bouton; SSR, subsynaptic reticulum. Bar, $500 \mathrm{~nm}$ (a and b); $250 \mathrm{~nm}(\mathbf{c}-\mathbf{e})$.

wall muscle extracts were not present in $n w k^{2}$ or $n w k^{2} / D f$ body wall muscle extracts, even though similar intensities of $\alpha$ Tubulin bands were detected. At least two abundant alternate splicing NWK variants are present in synapses in Drosophila larval NMJs. These precise and comparative localization patterns of Nwk reticulum suggest that NWK may be important for regulating synapse sizes and the trafficking of SV pools.

\section{Localization of Nwk at synapse edges and reserve SVs were revealed by immuno-EM}

Anti-Nwk immuno-EM analysis was performed to obtain further information regarding subsynaptic localization patterns of Nwk at synapses (Figure 2). Most silver- and gold-enhanced anti-Nwk particles were distributed at the edge of synapses $(46.2 \%)$ or within SV pools (44.6\%) in Type 1b synaptic boutons (Table 1 and Figure 2a). Two different types of SV pools at the presynaptic terminals of Drosophila NMJs have been suggested. ${ }^{26,43,44}$ The SVs involved with exocytosis present near the T-AZs are called the RRP. ${ }^{45}$ In contrast, SVs that are broadly distributed inside synaptic boutons are called the RP. ${ }^{26,43,45}$ At the EM levels, SVs near the T-AZs were considered as RRP. ${ }^{44}$ Therefore, the localization of Nwk particles in either the RRP or RP was further characterized. In this study, SVs present within $200 \mathrm{~nm}$ from the stub of T-AZs were considered to be in the RRP and were otherwise considered be in the RP. Among the 29 particles localized at the
SV pools, 5 particles were present in the RRP and 24 particles in the RP (Figures $2 \mathrm{c}-\mathrm{e}$ and Table 1). Synaptic terminals of Type Ib boutons in $n w k^{2}$ NMJs processed simultaneously with CS NMJs showed significantly reduced numbers of NWK particles (Figure 2b). Even though three times more thin sections obtained from $n w k^{2}$ NMJs than those of CS NMJs were examined, only three Nwk particles were observed at the presynaptic terminals and five particles at the postsynaptic terminals or muscles (Table 1). These immuno-EM results reinforced the subsynaptic distribution patterns of NWK at the edge of synapses and SV pools and suggesting possible roles of NWK in regulating synapse stability and transmission.

Synergistic phenotypes in the bouton numbers and branch extension in eag $^{1}$;nwk ${ }^{2}$ NMJs

Consistent with localization patterns of Nwk at the presynaptic terminals of Type 1 glutamtergic NMJs, several morphological phenotypes, including increased numbers of synaptic boutons, extended length of synaptic branches and reduced sizes of synaptic bouton area, were observed from $n w k^{2}$ null mutant NMJs when those phenotypes from CS NMJs were compared (Figures $3 \mathrm{a} 1,3 \mathrm{~b} 1$ and $31-3 \mathrm{k}$ ). The numbers of synaptic boutons in $n w k^{2}$ NMJs were significantly increased compared with those in CS NMJs (CS, $112.0 \pm 3.27 ; n w k^{2}, 148.2 \pm 3.22 ; n w k^{2} / D f$, $150.1 \pm 4.63$ ) (Figure 3i). The increased numbers of synaptic boutons have been considered evidence of activity-dependent 
Table 1 Localization of Nwk proteins at Drosophila Type I glutamatergic synaptic boutons

\begin{tabular}{|c|c|c|c|c|}
\hline \multirow[t]{2}{*}{ Location of Nwk particles } & \multicolumn{2}{|c|}{ Canton-S boutons } & \multicolumn{2}{|c|}{$n w k^{2}$ boutons } \\
\hline & No. of Nwk particles & $\%$ & No. of Nwk particles & $\%$ \\
\hline Edge of synapses & 30 & 46.2 & 2 & 25.0 \\
\hline T-shaped active zone & 1 & 1.5 & 0 & 0 \\
\hline RRPa & 5 & 8.0 & 0 & 0 \\
\hline Outside synaptic boutons (muscle sarcoplasm) & 3 & 4.6 & 5 & 62.5 \\
\hline Total & 65 & 100.0 & 8 & 100.0 \\
\hline
\end{tabular}

Abbreviations: Nwk, Nervous wreck; RP, reserve SV pool; RRP, readily releasable SV pool. Note that 22 thin sections from Canton-S boutons and 72 thin sections from $n w k^{2}$ null mutant boutons were examined.

asVs localized within $200 \mathrm{~nm}$ from the stub of T-AZs were considered the RRP.

bSV localized farther than $200 \mathrm{~nm}$ from the stub of T-AZs were considered the RP.
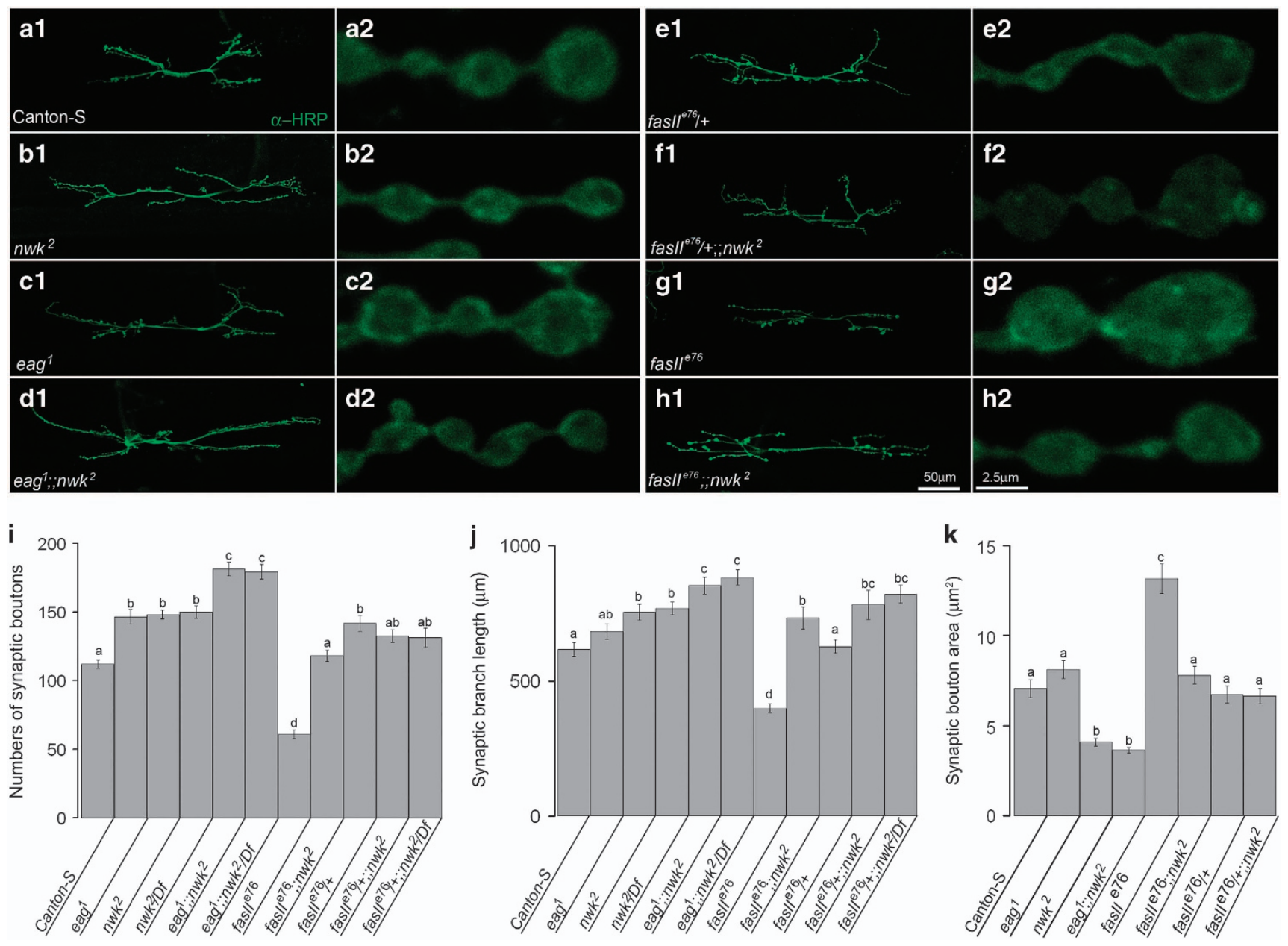

Figure 3 Altered synaptic morphology in $\mathrm{eag}^{1}$, fas//e76 and $n w k^{2}$ single- or double-mutant NMJs. Gross NMJ morphologies or synaptic bouton structures of CS (a1 and $\mathbf{a} 2), n w k^{2}$ (b1 and b2), eag ${ }^{1}$ (c1 and c2), eag ${ }^{1} ; n w k^{2}$ (d1 and d2), fas//e76/+ (e1 and e2), fas//p76/+;;nwk ${ }^{2}$

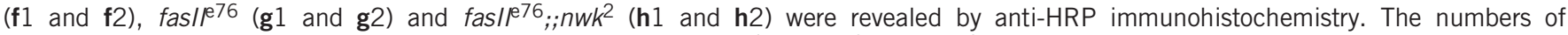
synaptic boutons (i), branch lengths (j) and bouton area (k) in eag ${ }^{1}$, fas $/ /^{76}$ and $n w k^{2}$ single- or double-mutant NMJs were compared with those of Canton-S. Characters above error bars indicate groups that are statistically identical. 

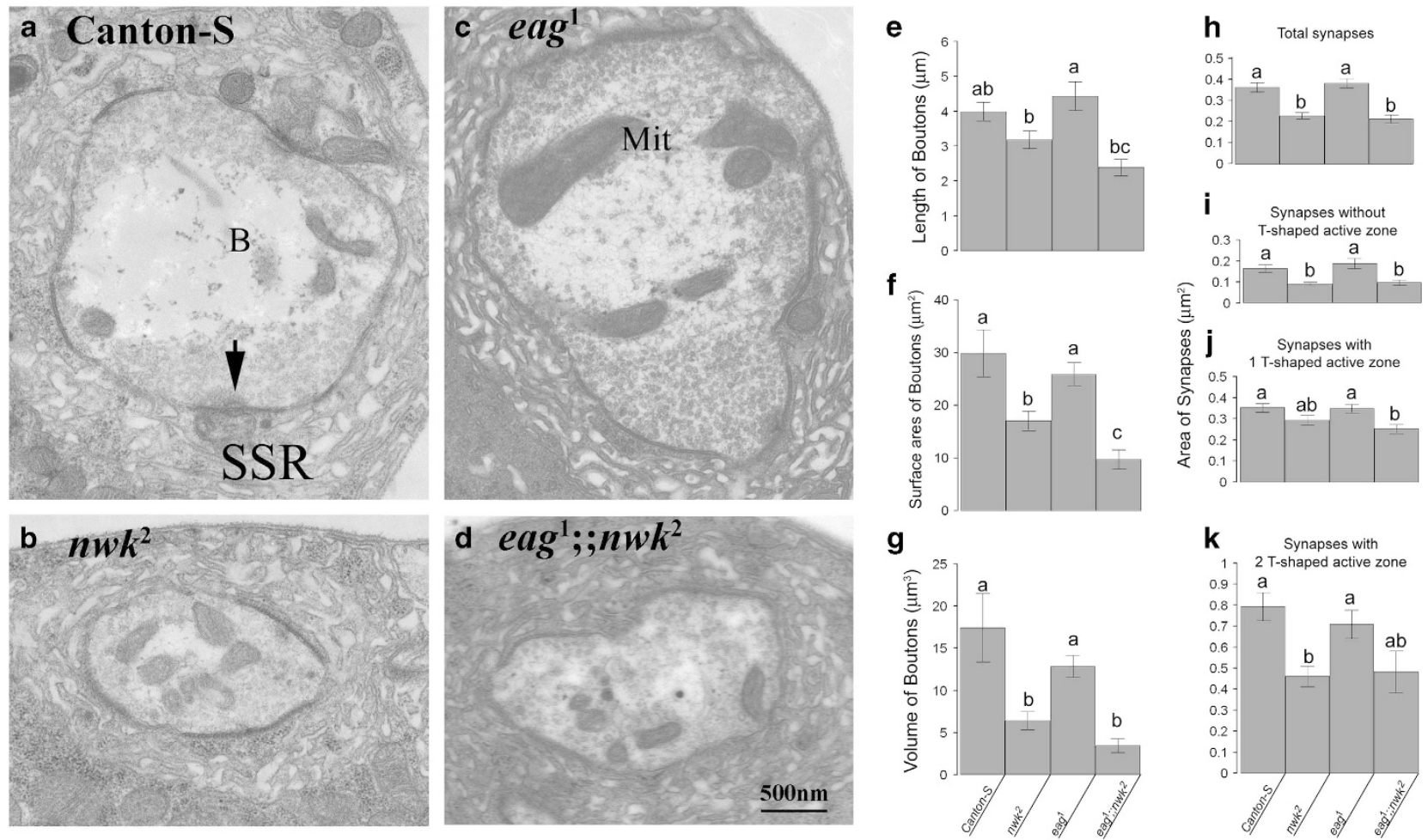

Figure 4 Ultrastructure of synaptic boutons in CS, $n w k^{2}$, eag ${ }^{1}$ or eag ${ }^{1} ; n w k^{2}$ NMJs. (a-d) Typical transmission electron microscope images taken from midline cross-section of $\mathrm{CS}(\mathbf{a}), n w k^{2}(\mathbf{b})$, eag ${ }^{1}(\mathbf{c})$ and eag $^{1} ; n w k^{2}$ (d) synaptic boutons. (e-g) Quantification results of the length (e), surface area (f) and volume (g) of synaptic boutons. (h-k) Areas of total synapses and of synapses without (i), with one (j) or with two (k) T-AZs. Black arrows indicate T-AZs in synapses. B, synaptic boutons; Mit, mitochondria; SSR, subsynaptic reticulum. Characters above error bars indicate groups that are statistically identical.

Hebbian plasticity. ${ }^{19}$ Mutations in eag, one of the voltage gated $\mathrm{K}^{+}$channels, induce hyperexcitability in Drosophila larval NMJs (Figure 3c1). ${ }^{19}$ The bouton number for eag ${ }^{1}$ NMJs $(146.6 \pm 6)$ was not statistically distinguishable from that for $n w k^{2}$ NMJs but was significantly increased compared with that for CS NMJs (Figures $3 \mathrm{~b} 1$ and i). Similar phenotypes between eag ${ }^{1}$ and $n w k^{2}$ mutant NMJs raised possibilities that eag and $n w k$ might be present in the same signaling pathway regulating the development of synaptic boutons. To test this possibility, eag ${ }^{1}$;; $n w k^{2}$ NMJs were generated and examined (Figures $3 \mathrm{~d} 1$ and i). eag ${ }^{1} ; n w k^{2}$ NMJs showed significantly increased bouton numbers (eag ${ }^{1} ; n w k^{2}, \quad 181.4 \pm 5.06 ;$ eag $\left.^{1} ; n n k^{2} / D f, \quad 179.5 \pm 5.56\right)$ compared with those in eag ${ }^{1}$ or $n w k^{2}$ NMJs (Figures $3 \mathrm{~d} 1$ and i). Synergistic phenotypes observed in eag ${ }^{1} ; n w k^{2}$ NMJs suggested that $e a g^{1}$ and $n w k^{2}$ are present in parallel or different signal transduction pathways regulating bouton numbers at Typel glutamatergic NMJs. This notion is supported by additional morphological phenotypes observed from $n w k^{2}$ NMJs, such as elongated synaptic branches or decreased areas of boutons, that were not observed in eag ${ }^{1}$ NMJs (Figures $3 j$ and $\mathrm{k}$ ). The synaptic branch lengths in $n w k^{2}$ or $n w k^{2} / D f$ NMJs $\left(n w k^{2}, 754.9 \pm 29.9 \mu \mathrm{m} ; n w k^{2} / D f, 768.8 \pm 23.8 \mu \mathrm{m}\right)$ were significantly increased compared with those of CS NMJs (CS, $617.4 \pm 25.1 \mu \mathrm{m})$. However, the synaptic branch length in eag ${ }^{1}$ NMJs $(683.3 \pm 28.7 \mu \mathrm{m})$ was not significantly different from those of either CS or $n w k$ mutant NMJs (Figure 3j). The length of eag and $n w k$ double-mutant NMJs (eag ${ }^{1} ; n w k^{2}$, $852.9 \pm 31.6 \mu \mathrm{m}$; $\left.\mathrm{eag}^{1} ; ; n w k^{2} / D f, 882.7 \pm 28.7 \mu \mathrm{m}\right)$ was significantly different from that of $n w k$ NMJs (Figures $3 \mathrm{~d} 1$ and $\mathrm{j}$ ). These results suggested that eag and $n w k$ may not act via the same signaling mechanisms regulating the numbers of synaptic boutons and NMJ length.

\section{Smaller synaptic boutons in $\mathrm{nwk}^{2}$ and eag $^{1} ; \mathrm{nnwk}^{2} \mathrm{NMJs}$} Another morphological phenotype in $n w k^{2}$ NMJ was reduced bouton sizes. Bouton sizes in $n w k^{2}$ NMJs $\left(4.095 \pm 0.225 \mu \mathrm{m}^{2}\right.$, $n=124$; Figures $3 \mathrm{~b} 2$ and $\mathrm{k}$ ) were significantly smaller than those of CS $\left(7.051 \pm 0.499 \mu \mathrm{m}^{2}, n=103\right.$; Figures $3 \mathrm{a} 2$ and $\left.\mathrm{k}\right)$ or eag $^{1}$ NMJs $\left(8.127 \pm 0.507 \mu \mathrm{m}^{2}, n=103\right.$; Figures $3 \mathrm{c} 2$ and $\left.\mathrm{k}\right)$ but similar to those of eag $; ; n w k^{2}$ NMJs $\left(3.650 \pm 0.154 \mu \mathrm{m}^{2}, \quad n=154\right.$; Figures $3 \mathrm{~d} 2$ and $\mathrm{k}$ ). Consistently, typical transmission EM images taken from across the midlines of boutons in CS, $n w k^{2}$, eag ${ }^{1}$ or eag $; ; n w k^{2}$ NMJs showed that bouton sizes in $n w k^{2}$ or eag $; ; n w k^{2}$ were significantly smaller than those in CS or eagl (Figures 4a-d). Further morphometric analysis by based on serial transmission EM images revealed that the average synaptic bouton length in $n w k^{2}$ NMJs $(3.18 \pm 0.25 \mu \mathrm{m}, n=9)$ was similar to those in CS NMJs $(3.99 \pm 0.26 \mu \mathrm{m}, n=7)$ but significantly smaller than those of eag ${ }^{1}$ NMJs $(4.43 \pm 0.41 \mu \mathrm{m}, n=7$; Figure 4e). The bouton surface area in $n w k^{2} \mathrm{NMJs}\left(17.04 \pm 1.87 \mu \mathrm{m}^{2}\right)$ was significantly decreased, $<70 \%$ of CS $\left(29.90 \pm 4.46 \mu \mathrm{m}^{2}\right)$ or $<60 \%$ of eag ${ }^{1}$ $\left(25.93 \pm 2.19 \mu \mathrm{m}^{2}\right)$ (Figure 4f). The bouton volume in $n w k^{2} \mathrm{NMJs}$ 

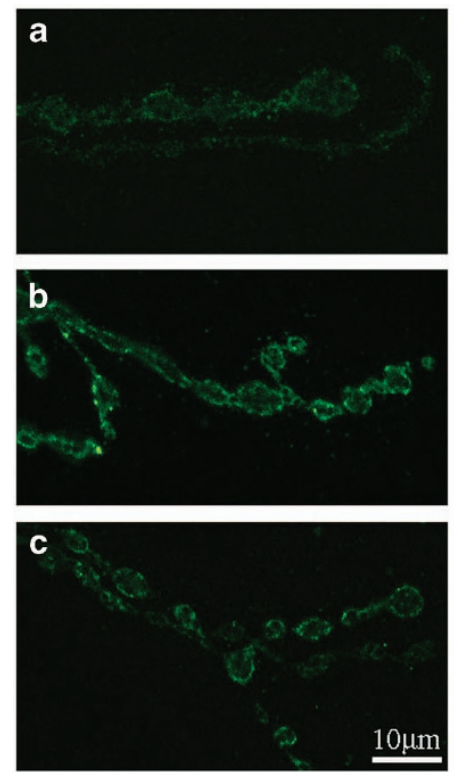

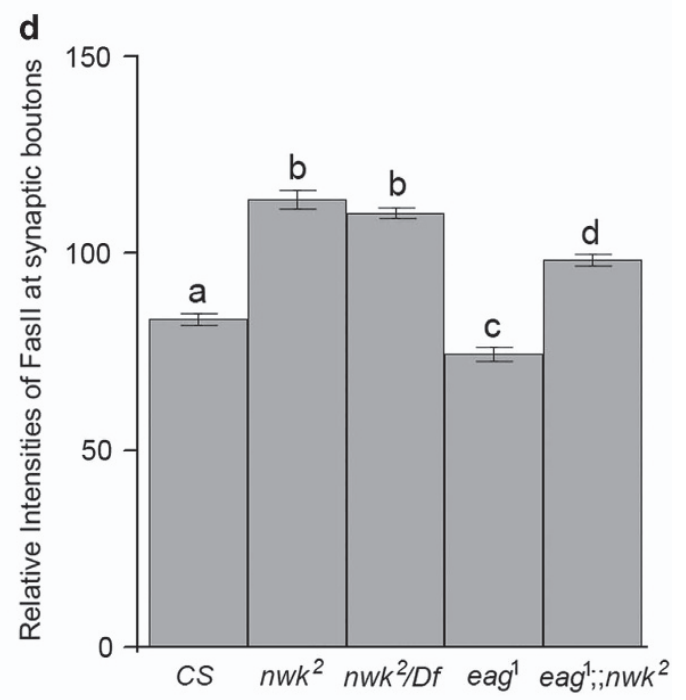

Figure 5 Increased Fasll localization at synapses and boutons in nwk NMJs. (a-c) Single-slice confocal images taken from several synaptic boutons of CS (a), $n w k^{2}$ (b) or $n w k^{2} / D f(\mathbf{c})$ larval NMJs. Increased Fasll levels were observed in nwk mutant NMJs (b and c). Relative intensities of anti-Fasll immunoreactivity at synaptic boutons of each genotype are compared (d). Characters above error bars indicate groups that are statistically identical.

$\left(6.39 \pm 1.09 \mu \mathrm{m}^{3}\right)$ was reduced to $<40 \%$ that in CS $\left(17.37 \pm 4.07 \mu \mathrm{m}^{3}\right) \quad$ or $\quad<50 \%$ that in eag ${ }^{1} \quad \mathrm{NMJs}$ $\left(12.81 \pm 1.3 \mu \mathrm{m}^{3}\right)$ (Figure $4 \mathrm{~g}$ ). Compared with $n w k^{2}$ NMJs, the bouton length and volume in $e a g^{1} ; ; n w k^{2}$ NMJs were statistically identical, but the average bouton surface area (length $=2.39 \pm 0.24 \mu \mathrm{m}, \quad$ surface $\quad$ area $=9.74 \pm 1.81 \mu \mathrm{m}^{2}$, volume $=3.38 \pm 0.82 \mu \mathrm{m}^{3}, n=9$ ) was not (Figures $4 \mathrm{e}-\mathrm{g}$ ). These results suggested that synaptic boutons in $e a g^{1} ; ; n w k^{2}$ might be more spherical compared with the more elliptical boutons in $n w k^{2}$. The normal development of sizes and shapes of synaptic boutons required the expression of Nwk.

\section{Reduced synapse areas in $\mathrm{nwk}^{2}$ and eag ${ }^{1} ; \mathrm{nwk}^{2}$ synaptic boutons}

Synapses are electron-dense parallel membrane structures usually harboring T-AZs surrounded by SVs at the presynaptic terminals (Figures $4 \mathrm{a}-\mathrm{d}$ ). The area of total synapses in CS $\left(0.364 \pm 0.022 \mu \mathrm{m}^{2}, n=133\right)$ was similar to that in eag ${ }^{1}$ $\left(0.383 \pm 0.023 \mu \mathrm{m}^{2}, n=113\right)$. In addition, the average total synaptic area was similar in $n w k^{2}\left(0.229 \pm 0.015 \mu \mathrm{m}^{2}, n=83\right)$ and eag ${ }^{1} ; n w k^{2}$ boutons $\left(0.213 \pm 0.019 \mu \mathrm{m}^{2}, \quad n=64\right)$, even though they were significantly smaller than those in CS or eag ${ }^{1}$ boutons (Figure $4 \mathrm{~h}$ ). Previous studies in synapses in Drosophila larval Type $1 \mathrm{~b}$ boutons suggested that synapses are ever changing structures undergoing emerging, strengthening, maturing, weakening or other processes. Studies in brp (Drosophila homolog of human active zone protein ELSK/CAST/ERC) loss-of-function mutants showed that T-AZs at synapses were essential for maintaining activitydependent release of SVs, rather than spontaneous release of SVs. ${ }^{39,40}$ These studies suggested that synapses without a T-AZ may be silent, while one or more T-AZs may be active synapses undergoing activity-dependent SV exocytosis. Therefore, the synapses in each genotype were further divided by the numbers of T-AZs present. In this analysis, $25 \%$ of CS synapses, $22 \%$ of $n w k^{2}$ synapses, $18 \%$ of eag ${ }^{1}$ synapses and $34 \%$ of $e a g^{1} ; ; n w k^{2}$ synapses lacked a T-AZ and were considered silent synapses, while $63 \%$ of CS synapses, $65 \%$ of $n w k^{2}$ synapses, $64 \%$ of eag ${ }^{1}$ synapses and $59 \%$ of eag ${ }^{1} ; n w k^{2}$ synapses were with one T-AZ. In addition, $11 \%$ of CS synapses, $12 \%$ of $n w k^{2}$ synapses, $19 \%$ of eag synapses and $6 \%$ of eag ${ }^{1} ; n n k^{2}$ synapses had two or more T-AZs. The areas of silent synapses in $n w k^{2}\left(0.090 \pm 0.009 \mu \mathrm{m}^{2}\right)$ and eag $; ; n w k^{2}$ $\left(0.096 \pm 0.012 \mu \mathrm{m}^{2}\right)$ boutons were $<50-60 \%$ of those in CS $\left(0.163 \pm 0.017 \mu \mathrm{m}^{2}\right)$ and eag $\left(0.187 \pm 0.024 \mu \mathrm{m}^{2}\right)$ boutons (Figure 4i). However, the area of synapses with one T-AZ in eag ${ }^{1} ; n w k^{2}\left(0.252 \pm 0.022 \mu \mathrm{m}^{2}\right)$ boutons was only significantly different from those in CS $\left(0.352 \pm 0.02 \mu \mathrm{m}^{2}\right)$ or eag ${ }^{1}$ $\left(0.348 \pm 0.02 \mu \mathrm{m}^{2}\right)$ boutons (Figure $\left.4 \mathrm{j}\right)$. The areas of synapses with one T-AZ in $n w k^{2}\left(0.293 \pm 0.022 \mu \mathrm{m}^{2}\right)$ were not significantly different from those of CS, eag ${ }^{1}$ or $e a g^{1} ; ; n w k^{2}$. The areas of synapses with two T-AZs in $n w k^{2}$ boutons were significantly reduced, to $\sim 60 \%$ of those in CS or eag ${ }^{1}$ boutons (Figure $4 \mathrm{k}$, CS, $\quad 0.793 \pm 0.0669 \mu \mathrm{m}^{2} ; \quad n w k^{2} ; \quad 0.462 \pm 0.0486 \mu \mathrm{m}^{2} ; \quad$ eag ${ }^{1}$, $0.709 \pm 0.0663 \mu \mathrm{m}^{2} ; \quad$ eag $\left.; ; n w k^{2} ; \quad 0.482 \pm 0.0989 \mu \mathrm{m}^{2}\right)$. These EM morphometry results showed that Nwk has important roles in maintaining synapses at the proper sizes, and they explain why $n w k^{2}$ mutants showed reduced evoked junctional current in a previous study. ${ }^{31}$

FasII levels at synaptic boutons were increased at nwk NMJs Another phenotype observed from NMJs in hyperexcitability mutant is decreased FasII localization at boutons. ${ }^{20}$ Downregulation of FasII at boutons by increased neuronal activities, 
hypomorphic mutations in fasII or activation of mitogenactivated protein kinase-RAS signaling in NMJs resulted in increased numbers of boutons. ${ }^{46}$ Given that Nwk acts via a signaling mechanism different from that of canonical Hebbian plasticity, FasII localization at boutons of $n w k^{2}$ NMJs might be different from those of boutons of eag ${ }^{1}$ NMJs. Indeed, FasII localization at boutons in $n w k^{2}$ NMJs was significantly increased compared with boutons in CS or eag ${ }^{1}$ NMJs (Figures $5 \mathrm{a}-\mathrm{c}$ ). Relative FasII immunoreactivity at the NMJs in $n w k^{2}$ or $n w k^{2} / D f$ mutants was $120.3 \pm 2.6$ or $119.4 \pm 2.1$, respectively, an increase of $\sim 40 \%$ compared with those in CS $(86.3 \pm 1.3)$. FasII levels in $e^{1} g^{1} ; n w k^{2}$ NMJs $(98.3 \pm 1.5)$ were increased by $\sim 30 \%$ compared with those in eag ${ }^{1}$ NMJs $(74.4 \pm 1.8)$ and decreased by $\sim 20 \%$ compared with those in $n w k^{2}$ or $n w k^{2} / D f$ (Figure $4 \mathrm{~d}$ ). These adjusted FasII levels in $e a g^{1} ; n w k^{2}$ NMJs reinforced our hypothesis that Nwk is a component of a signaling mechanism that is different from canonical Hebbian plasticity.

\section{Genetic interaction between Nwk and FasII in regulating synaptic morphology}

The synaptic bouton number $(61.0 \pm 3.33)$ and branch length $(399.6 \pm 17.0 \mu \mathrm{m})$ in fasII ${ }^{e 76} \mathrm{NMJ}$ were $<50 \%$ of those in CS or $n w k^{2}$ NMJs (Figures 3g1). However, the synaptic boutons of fasII ${ }^{e 76}\left(13.18 \pm 0.93 \mu \mathrm{m}^{2}, n=82\right)$ were twice as large as those of CS (Figures 3g2 and k). Further, fasII $I^{\mathrm{e} 6} /+\mathrm{NMJs}$ expressed FasII protein at $\sim 55 \%$ of the level observed in CS NMJs and had similar average numbers of synaptic boutons $(141.8 \pm 5.64)$ as $e a g^{1}$ and $n w k^{2}$ NMJs (Figures $3 \mathrm{e} 1$ and i). The branch length and bouton area in fasI $\mathrm{I}^{\mathrm{e} 6} / \mathrm{NMJ}$ sere not significantly different from those of CS or eag ${ }^{1}$ NMJs (Figures 3e1). These results suggested that reduced FasII at NMJs is enough to induce increased bouton numbers but not sufficient to extend the length of synaptic branches. Increased FasII localization at boutons of $n w k^{2}$ NMJs (Figure 5) and compensatory localization patterns between Nwk and FasII at boutons of CS NMJs (Figure 1c) suggested that Nwk and FasII may have complementary roles in regulating synaptic plasticity. To gain insight into unknown mechanisms underlying synaptic plasticity by FasII and Nwk, FasII protein levels in $n w k^{2}$ NMJs were genetically regulated by recombining fasII ${ }^{276}$ mutant chromosomes. When FasII expression was reduced to $10 \%$ of CS, the numbers of synaptic boutons and bouton midline areas in fasII ${ }^{e 76} ; n n w k^{2}\left(118.1 \pm 4.22 ; 729 \pm 0.471 \mu \mathrm{m}^{2}, n=102\right)$ were not significantly different from those of CS (Figures 3h1). These results suggested that fasII $I^{\mathrm{e} 6}$ and $n w k^{2}$ mutations mutually suppressed the opposing phenotypes when $10 \%$ of the normal level of FasII was expressed. Only the length of synaptic branches of $f a s I I^{e 76} ; n w k^{2}(732.8 \pm 40.4 \mu \mathrm{m})$ was similar to those of $n w k^{2}$ NMJs. When FasII expression was reduced to $55 \%$ of CS, the bouton numbers of fasII ${ }^{e 76} /+; n w w k^{2}(132.6 \pm 4.73)$ and fasII $I^{e 76} /+; n w k^{2} / D f(131.4 \pm 6.92)$ NMJs were similar to those of fasII $I^{76} /+$ and $n w k^{2}$ mutants (Figures $3 \mathrm{fl}$ and $\mathrm{i}$ ). However, the synaptic branch lengths of $f a s I I^{776} /+; n w w k^{2}(781.0 \pm 54.8 \mu \mathrm{m})$ and $f a s I I^{776} /+; n w k^{2} / D f(821.4 \pm 34.1 \mu \mathrm{m})$ were similar to those of $n w k^{2}$ mutants. In addition, the bouton midline areas of
fasII $I^{e 76} /+; n w k^{2} \quad\left(6.638 \pm 0.41 \mu \mathrm{m}^{2}, \quad n=128\right)$ were similar to those of fasII $I^{e 76} /+$ (Figures $3 \mathrm{f} 2$ and $\mathrm{k}$ ).

Our NMJ morphology analysis results suggested that three morphological aspects examined in this study could be regulated by different signaling mechanisms. We can suggest that FasII is downstream of Nwk in regulating the numbers and sizes of synaptic boutons but either is upstream in the regulation of synaptic branch length or does not participate in this process. Further studies with other mutants showing altered morphologies will reveal more details of the signaling mechanisms.

\section{nwk synapses showed impaired RRPs at T-AZs}

The localization of Nwk with SVs at the confocal microscopic level (Figure 1) and EM level (Figure 2) suggested that Nwk may have important roles in regulating SV trafficking or transmission. Electrophysiological studies showed that the quantal contents of $n w k^{2}$ NMJs were $\sim 50 \%$ less than those of CS, although the amplitude of miniature excitatory junctional currents or quantal sizes were slightly increased without changes in frequency of miniature excitatory junctional currents. $^{31}$ Even though the $\sim 50 \%$ decrease in the numbers of synapses and T-AZs in $n w k^{2}$ boutons may contribute to reduced synaptic transmission in $n w k^{2} \mathrm{NMJs}$, the numbers of synaptic boutons in $n w k^{2}$ NMJs were increased by $\sim 40 \%$. These results suggested that other defects caused by loss of Nwk at synaptic terminals may exist. Thus we further examined whether SV pools in $n w k^{2}$, eag $g^{1}$ or eag $; ; n w k^{2}$ boutons were altered.

First, we examined whether Mab-Synapsin signals in $n w k^{2}$ boutons were altered (Figures $6 \mathrm{a}-\mathrm{d}$ ). When $n w k^{2}$, eag ${ }^{1}$ or $e a g^{1}$;; $n w k^{2}$ boutons were compared with CS, only $n w k^{2}$ boutons appeared to have reduced Synapsin immunoreactivity. eag ${ }^{1}$ boutons showed stronger signals and $e a g^{1} ; n n k^{2}$ boutons showed signals similar to those of CS. These results suggested that Nwk may be critical for maintaining normal SV pools at the presynaptic terminals.

Next, we examined whether RRP and RP were altered in $n w k^{2}$ boutons by performing EM (Figures $6 \mathrm{e}-\mathrm{h}$ ). In this study, the RRP and RP were defined as SVs within $200 \mathrm{~nm}$ or at $200-400 \mathrm{~nm}$, respectively, from the stub of T-AZs. The numbers of SVs in the RRP $(6.44 \pm 0.27)$ or RP $(10.83 \pm 0.53)$ in $n w k^{2}$ were significantly less than those of CS (RRP, $11.49 \pm 0.28 ; \quad R P, \quad 24.115 \pm 0.77)$. The RRPs of eag ${ }^{1}$ $(12.72 \pm 0.38)$ and $e^{2} g^{1} ; n w k^{2}(12.43 \pm 0.30)$ were significantly increased compared with that of CS. However, only the RP of eag $^{1}(28.21 \pm 0.91)$ was significantly increased compared with that of CS. The RP of $e a g^{1} ; ; n w k^{2}(23.26 \pm 0.82)$ was similar to that of CS (Figure 6i). These results suggested that Nwk is critical for maintaining the RRP and RP. In addition, the reduction in SVs induced by the loss of Nwk at the presynaptic terminals might be offset by increased neural activity, which is known to strengthen synaptic connections.

\section{Nwk formed a protein complex with DSypt1 and Synapsin} Reduced Synapsin immunoreactivities together with reduced RRP and RP at $n w k$ mutant synapses suggested that NWK and 

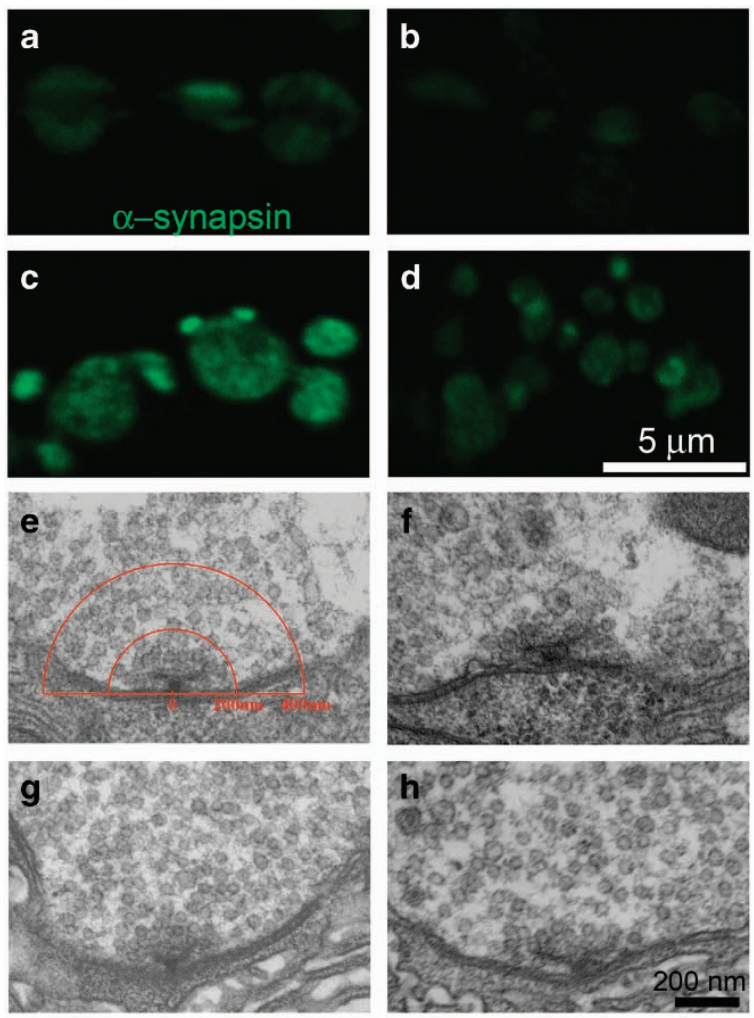
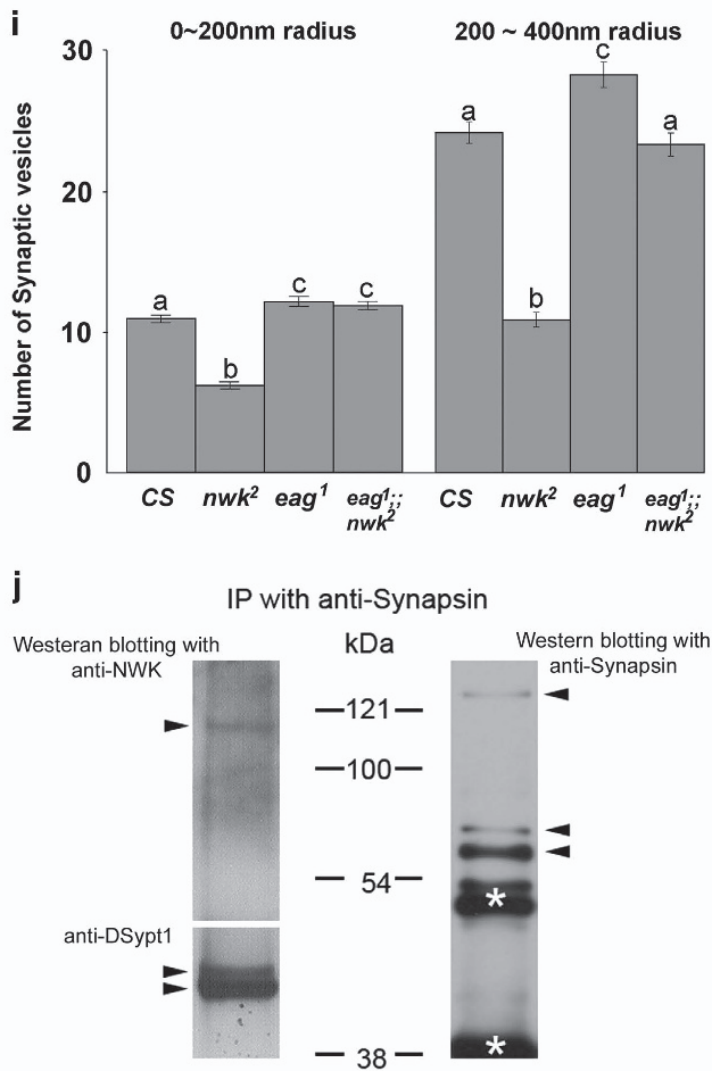

Figure 6 Synapsin immunoreactivity and SV numbers were significantly reduced in $n w k^{2}$ mutant synaptic boutons. (a-d) Mab-Synapsin immunoreactivity in $n w k^{2}$ mutant synaptic boutons (b) appeared to be weaker than CS (a), eag ${ }^{1}$ (c) or eag ; ;nwk ${ }^{2}$. (e-f) Ultrastructural analysis of SVs near T-AZs showed fewer SVs in $n w k^{2}$ (f), eag ${ }^{1}(\mathbf{g})$ or $\operatorname{eag}^{1} ;$; $\boldsymbol{k}^{2}(\mathbf{h})$ compared with CS (d). (i) The SV numbers in RRP $(0-200 \mathrm{~nm})$ or RP $(200-400 \mathrm{~nm})$ from a T-AZ in $n w k^{2}$ mutant synaptic boutons were significantly reduced. (j) Mab-Synapsin coprecipitated with Nwk, DSypt1 and Synapsin from protein extracts from larval body wall muscle preparations. Characters above error bars indicate groups that are statistically identical.

Synapsin may present at the same protein complexes. To investigate this possibility, we performed Mab-Synpasin immunoprecipitation and proved precipitates with rabbit anti-Nwk or anti-DSypt1 antibodies (Figure 6j). There were clear one Nwk and two DSyptl bands when Mab-Synapsin precipitates were separated. Consistent with a previous report, ${ }^{47}$ three Synapsin bands were clearly detected. These results suggested that NWK formed a protein complex with DSypt1 and Synapsin and regulates SV trafficking and transmission.

\section{D HVEM tomography revealed T-AZ architectures of CS and $\mathrm{nwk}^{2}$ synapses at high resolution}

To investigate SV localization patterns and synaptic architecture at three dimensions, CS and $n w k^{2}$ synapses in Type $1 \mathrm{~b}$ boutons were analyzed by 3D HVEM tomography. Examples of unprocessed HVEM images of CS and $n w k^{2}$ synapses are shown in Figures $7 \mathrm{a}$ and b. Using 121 tilted images, 3D ultrastructures of synapses (Figures $7 \mathrm{c}$ and $\mathrm{d}$ ) and T-AZs (Figures $7 \mathrm{e}$ and $\mathrm{f}$ ) of $\mathrm{CS}$ and $n w k^{2}$ were generated by IMOD. ${ }^{37}$ Because fewer SVs with enlarged sizes were observed from $n w k^{2}$, we further quantified the 3D distribution patterns of SVs in RRPs. Consistent with conventional EM results (Figures 6e-h), significantly fewer SVs presented at RRP (CS, $\left.138.0 \pm 9.0 ; n w k^{2}, 91.0 \pm 6.8, P<0.01\right)$. The graphs showing SVs numbers based on distances from the stub of T-AZs suggested that no SVs were docked to T-AZs in $n w k^{2}$. However, more SVs localized from 100 to $140 \mathrm{~nm}$ from T-AZs in $n w k^{2}$ than in CS. In addition, SV size distribution results (Figure 7i) revealed that SVs in $n w k^{2}$ were significantly larger than those in CS $\left(n w k^{2}, 42.69 \pm 0.45 ;\right.$ CS, $\left.39.40 \pm 0.315 \mathrm{~nm}, P<0.001\right)$. The $3 \mathrm{D}$ ultrastructural differences at synapses and T-AZs between CS and $n w k^{2}$ were clearly visualized by 3D rotation videos (Supplementary Video Clips 1). Significantly fewer but enlarged SVs were present in the RRPs in $n w k^{2}$ synapses.

\section{DISCUSSION}

In order to investigate the unknown in vivo functions of proteins containing $\mathrm{SH} 3$ and F-BAR domains in synaptic plasticity and transmission, we took advantage of Drosophila NMJs and available $n w k$ mutants. Nwk is a Drosophila homolog of MEGAP, one of the proteins responsible for mental retardation in humans; Nwk was initially discovered by temperature-sensitive paralysis behavior of adult flies. ${ }^{31}$ Although genetic and physical interactions between Nwk and components of endocytosis, such as Dap160 and Dynamin, ${ }^{48}$ and actin cytoskeleton regulators, such as Wiskott-Aldrich 

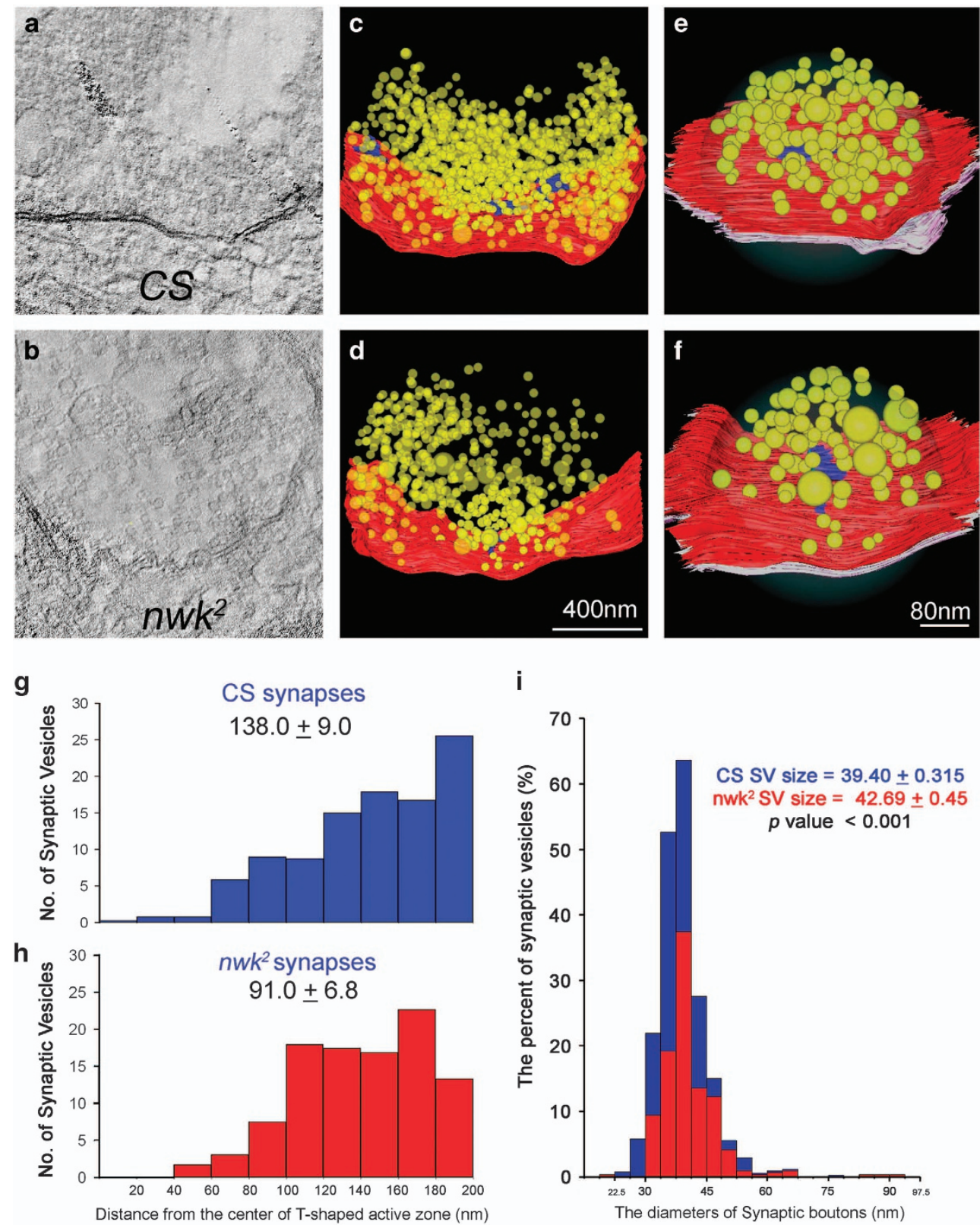

Figure 7 HVEM analysis of nwk mutant synapses reinforced altered SV sizes and distribution. Representative HVEM photographs taken from CS (a) or $n w k^{2}$ (b) synapses before processing with IMOD. 3D images of synapses and active zones in CS (c) or $n w k^{2}$ (d). The numbers of SVs from T-AZs in CS (g) or $n w k^{2}(\mathbf{h})$ synapses were quantified. The ratio of SVs in CS (blue) and $n w k^{2}$ (red) based on their diameters (i) using 400 SVs from CS and 318 SVs from $n w k^{2}$.

syndrome protein $^{31}$ and $\mathrm{CDC} 42,{ }^{49}$ have been reported, the localization patterns of Nwk at synaptic boutons appear to be different from localization patterns of the abovementioned proteins. Thus we hypothesized that Nwk may directly or indirectly interact with other synaptic or cytoskeletal components. In addition, by performing immunohistochemistry to confirm partial colocalization of Nwk with DAP160 (Figure $1 \mathrm{j}$ ), ${ }^{48}$ we also observed via confocal microscopy that Nwk partially colocalized (Figures le and f) and formed protein complexes (Figure 6j) with Synapsin and DSypt1. Furthermore, we found that Synapsin immunoreactivities at $n w k$ synaptic boutons were significantly reduced (Figures 6a-d). These findings are highly intriguing because recent studies have shown that Synapsin, the most abundant presynaptic phosphoprotein in Drosophila, has a pivotal role in regulating SV trafficking at synaptic terminals by forming protein complexes with Dap $160^{50}$ in glutamatergic NMJs. The defects of RRP and $\mathrm{RP}$ in $n w k$ mutant synapses were further confirmed by performing transmission EM analysis (Figures 6e-i) and HVEM 3D tomography (Figures $7 \mathrm{a}-\mathrm{i}$ ). Thus it is possible that Nwk is actively participating in SV trafficking at RRP and RP via interactions with Synapsin, DSypt1 (Figure 6i), Dap160, ${ }^{50}$ Dynamin $^{48}$ and other interacting proteins that have yet to be identified. 
Another phenotype in $n w k$ loss-of-function mutants that was able to be identified by this study's use of HVEM 3D tomography was that of enlarged SVs (Figures 7a-i). Because we used $1 \mu \mathrm{m}$ thick sections for examining size and distribution of SVs at $n w k$ mutant and control synapses and as $20 \mathrm{~nm}$ gold particles were used to create a list of fiducial points and size standards for SVs, our HVEM 3D analysis results did not contain biased data originating from measuring diameters of sectioned SVs when $50-100 \mathrm{~nm}$ of thin sections were used for conventional transmission EM analysis as described by Kim et al. ${ }^{51}$ Recently, it has been shown that an F-BAR domain in Nwk binds to and remodels membranes to form concave membrane structures. ${ }^{52}$ Therefore, it is possible that Nwk may regulate SV recycling both directly, by interacting with synaptic membranes, and indirectly, through its binding partners, such as Synapsin, DSypt1, Dap160 and Dynamin. Thus loss of Nwk at synapses might induce enlarged SVs compared with control synapses.

In addition to regulating SV size and trafficking, our NMJ and synapse morphometric analysis results provided several lines of evidence supporting that Nwk regulates synaptic plasticity of glutamatergic NMJs in a manner different from activity-dependent Hebbian plasticity. Previous studies have shown one of the classical examples of Hebbian plasticity, in which increased neuronal activity in NMJs reduces FasII localization at synaptic boutons, resulting in both an increase in the number of synaptic boutons and branch elongation. ${ }^{19,22}$ However, the findings of this study, which supported that genetically increased neuronal activity in $n w k^{2}$ mutant NMJs resulted in significantly more synaptic boutons and branch elongations (Figure 3) and that the levels of FasII in $n w k^{2}$ mutants were significantly higher than those of CS (Figure 5), suggested that Nwk may exist in a different signaling pathway from that of Hebbian plasticity.

The evidence presented in this study is important because most psychiatric and neurological diseases, ranging from autism and mental retardation to dementia, are reported to be accompanied by altered synaptic structures and functions. ${ }^{1}$ The association of MEGAP and mental retardation has been revealed in the process of identifying genes that are linked with distal chromosome $3 p$ deletion syndrome (3p-syndrome) patients with severe mental retardation. ${ }^{3,53}$ Similar to Nwk, MEGAP expression was observed in the embryonic central nervous systems of mice ${ }^{54}$ and high expression was found in the hippocampus, amygdala, piriform cortex, posterior parietal associative area and the dentate gyrus of adult mouse brains. ${ }^{3}$ In addition, similar to Nwk, MEGAP binds strongly to the phosphoinositide-rich membrane and is involved in the formation of filopodia. ${ }^{52}$ In the synaptogenesis stage, MEGAP has an important role in synapse selection, and consequently, the loss of MEGAP function results in a decrease in the number of neuron spines. ${ }^{55}$ Thus, although it has not been elucidated to date whether MEGAP expresses at presynaptic or postsynaptic terminals or both, it is possible that MEGAP may regulate SV size and distribution in neuron spines at glutamatergic synapses of the mouse central nervous system, especially at the hippocampus, resulting in mental retardation phenotypes in human patients ${ }^{3}$ and mouse knockout models. ${ }^{55,56}$ Thus further studies in Nwk and MEGAP will reveal unknown molecular and cellular functions of SH3 and F-BAR domain proteins in synaptic plasticity and mental retardation.

\section{CONFLICT OF INTEREST}

The authors declare no conflict of interest.

\section{ACKNOWLEDGEMENTS}

The Korea Basic Science Institute kindly provided HVEM and IMOD analysis. This work was carried out with the support of the Cooperative Research Program for Agriculture Science \& Technology Development (Project title: Elucidation the health improvement effects of boiled silk worm larvae, Project No.: PJ010828012017), the Rural Development Administration and the Basic Science Research Program through the National Research Foundation of Korea (NRF) funded by the Ministry of Education, Science and Technology (No.

2015R1D1A3A01018497).

\section{PUBLISHER'S NOTE}

Springer Nature remains neutral with regard to jurisdictional claims in published maps and institutional affiliations.

1 van Spronsen M, Hoogenraad CC. Synapse pathology in psychiatric and neurologic disease. Curr Neurol Neurosci Rep 2010; 10: 207-214.

2 Turrigiano GG. The self-tuning neuron: synaptic scaling of excitatory synapses. Cell 2008; 135: 422-435.

3 Endris V, Wogatzky B, Leimer U, Bartsch D, Zatyka M, Latif F et al. The novel Rho-GTPase activating gene MEGAP/ srGAP3 has a putative role in severe mental retardation. Proc Natl Acad Sci USA 2002; 99: 11754-11759.

4 Jin P, Alisch RS, Warren ST. RNA and microRNAs in fragile $X$ mental retardation. Nat Cell Biol 2004; 6: 1048-1053.

5 Gabellini D, Green MR, Tupler R. When enough is enough: genetic diseases associated with transcriptional derepression. Curr Opin Genet Dev 2004; 14: 301-307.

6 Belmonte MK, Allen G, Beckel-Mitchener A, Boulanger LM, Carper RA, Webb SJ. Autism and abnormal development of brain connectivity. J Neurosci 2004; 24: 9228-9231.

7 Fang P, Lev-Lehman E, Tsai TF, Matsuura T, Benton CS, Sutcliffe JS et al. The spectrum of mutations in UBE3A causing Angelman syndrome. Hum Mol Genet 1999; 8: 129-135.

8 Zoghbi HY. Postnatal neurodevelopmental disorders: meeting at the synapse? Science 2003; 302: 826-830.

9 Turrigiano GG, Nelson SB. Homeostatic plasticity in the developing nervous system. Nat Rev Neurosci 2004; 5: 97-107.

10 Vitureira N, Goda Y. The interplay between Hebbian and homeostatic synaptic plasticity. J Cell Biol 2013; 203: 175-186.

11 Toyoizumi T, Kaneko M, Stryker MP, Miller KD. Modeling the dynamic interaction of hebbian and homeostatic plasticity. Neuron 2014; 84: 497-510.

12 Desai NS. Homeostatic plasticity in the CNS: synaptic and intrinsic forms. J Physiol 2003; 97: 391-402.

13 Abbott LF, Regehr WG. Synaptic computation. Nature 2004; 431 : $796-803$.

14 O'Rourke NA, Weiler NC, Micheva KD, Smith SJ. Deep molecular diversity of mammalian synapses: why it matters and how to measure it. Nat Rev Neurosci 2012; 13: 365-379.

15 Koh YH, Gramates LS, Budnik V. Drosophila larval neuromuscular junction: molecular components and mechanisms underlying synaptic plasticity. Microsc Res Tech 2000; 49: 14-25.

16 Keshishian H. Is synaptic homeostasis just wishful thinking? Neuron 2002; 14: $481-482$ 
17 Packard M, Mathew D, Budnik V. Wnts and TGF- $\beta$ in synaptogenesis: old friends signaling at new places. Nat Rev Neurosci 2003; 4: 113-120.

$18 \mathrm{Koh} \mathrm{YH}$. Drosophila as a model organism for investigating molecular and cellular etiologies underlying complex neurological disorders in humans. J Asia Pac Entomol 2006; 9: 75-84.

19 Budnik V, Zhong Y, Wu CF. Morphological plasticity of motor axons in Drosophila mutants with altered excitability. J Neurosci 1990; 10: 3754-3768.

20 Schuster CM, Davis GW, Fetter RD, Goodman CS. Genetic dissection of structural and functional components of synaptic plasticity. I. Fasciclin II controls synaptic stabilization and growth. Neuron 1996; 17: 641-654.

21 Schuster CM, Davis GW, Fetter RD, Goodman CS. Genetic dissection of structural and functional components of synaptic plasticity. II. Fasciclin II controls presynaptic structural plasticity. Neuron 1996; 17: 655-667.

22 Davis GW, Schuster CM, Goodman CS. Genetic analysis of the mechanisms controlling target selection: target-derived Fasciclin II regulates the pattern of synapse formation. Neuron 1996; 19: 561-573.

23 Ljaschenko D, Ehmann N, Kittel RJ. Hebbian plasticity guides maturation of glutamate receptor fields in vivo. Cell Rep 2013; 3 : 1407-1413.

$24 \mathrm{Kim}$ E, Sheng M. PDZ domain proteins of synapses. Nat Rev Neurosci 2004; 5: 771-781.

25 Rizzoli SO, Betz WJ. Synaptic vesicle pools. Nat Rev Neurosci 2005; 6 : 57-69.

26 Kidokoro Y, Kuromi H, Delgado R, Maureira C, Oliva C, Labarca P. Synaptic vesicle pools and plasticity of synaptic transmission at the Drosophila synapse. Brain Res Brain Res Rev 2004; 47: 18-32.

27 Tejedor FJ, Bokhari A, Rogero O, Gorczyca M, Zhang J, Kim E et al. Essential role for dlg in synaptic clustering of Shaker $\mathrm{K}^{+}$channels in vivo. J Neurosci 1997; 17: 152-159.

28 Koh YH, Popova E, Thomas U, Griffith LC, Budnik V. Regulation of DLG localization at synapses by CaMKII-dependent phosphorylation. Cell 1999; 98: 353-363.

29 Thomas U, Kim E, Kuhlendahl S, Koh YH, Gundelfinger ED, Sheng M et al. Synaptic clustering of the cell adhesion molecule fasciclin II by discs-large and its role in the regulation of presynaptic structure. Neuron 1997; 19: 787-799.

30 Paradis S, Sweeney ST, Davis GW. Homeostatic control of presynaptic release is triggered by postsynaptic membrane depolarization. Neuron 2001; 30: 737-749.

31 Coyle I, Koh YH, Lee W-CM, Slind J, Fergestad T, Littleton JT et al. Nervous Wreck, an adaptor protein that interacts with WASP, regulates synapse growth in Drosophila. Neuron 2004; 41: 512-534.

$32 \mathrm{Kim}$ AY, Seo JB, Kim WT, Choi HJ, Kim S-Y, Morrow G et al. The pathogenic human Torsin $A$ in Drosophila activates the unfolded protein response and increases susceptibility to oxidative stress. BMC Genomics 2015; 16: 338

33 Lee DW, Seo JB, Ganetzky B, Koh YH. $\Delta$ FY mutation in human Torsin A induces locomotor disability and abberant synaptic structures in Drosophila. Mol Cells 2009; 27: 89-97.

34 Zhang B, Koh YH, Beckstead RB, Budnik V, Ganetzky B, Bellen HJ. Synaptic vesicle size and number are regulated by a Clathrin adaptor protein required for endocytosis. Neuron 1998; 21: 1465-1475.

35 Roos J, Kelly RB. Dap160, a neural-specific Eps15 homology and multiple SH3 domain-containing protein that interacts with Drosophila dynamin. J Biol Chem 1998; 273: 19108-19119.

36 Schneider CA, Rasband WS, Eliceiri KW. NIH Image to ImageJ: 25 years of image analysis. Nat Methods 2012; 9: 671-675.

37 Kremer JR, Mastronarde DN, McIntosh JR. Computer visualization of threedimensional image data using IMOD. J Struct Biol 1995; 116: 71-76.

38 Koh YH, Lehfield K, Ganetzky B. A Drosophila model of early-onset torsion dystonia suggests impairments in transforming growth factor $\beta$ signaling. Hum Mol Genet 2004; 13: 2019-2030.

39 Wagh DA, Rasse TM, Asan E, Hofbauer A, Schwenkert I, Durrbeck H et al. Bruchpilot, a protein with homology to ELKS/CAST, is required for structural integrity and function of synaptic active zones in Drosophila. Neuron 2006; 49: 833-844.
40 Kittel RJ, Wichmann C, Rasse TM, Fouquet W, Schmidt M, Schmid A et al. Bruchpilot promotes active zone assembly, $\mathrm{Ca}^{2+}$ channel clustering, and vesicle release. Science 2006; 312: 1051-1054.

41 Koh TW, Verstreken P, Bellen HJ. Dap160/Intersectin acts as a stabilizing scaffold required for synaptic development and vesicle endocytosis. Neuron 2004; 43: 193-205.

42 Roos J, Hummel T, Ng N, Klambt C, Davis GW. Drosophila Futsch regulates synaptic microtubule organization and is necessary for synaptic growth. Neuron 2000; 26: 371-382.

43 Delgado R, Maureira C, Oliva C, Kidokoro Y, Labarca P. Size of vesicle pools, rates of mobilization, and recycling at neuromuscular synapses of a Drosophila mutant, shibire. Neuron 2000; 28: 941-953.

44 Roche JP, Packard MC, Moeckel-Cole S, Budnik V. Regulation of synaptic plasticity and synaptic vesicle dynamics by the PDZ protein Scribble. J Neurosci 2002; 22: 6471-6479.

45 Alabi AA, Tsien RW. Synaptic vesicle pools and dynamics. Cold Spring Harb Perspect Biol 2012; 4: a013680.

46 Koh YH, Ruiz-Canada C, Gorczyca M, Budnik V. The Ras1-mitogenactivated protein kinase signal transduction pathway regulates synaptic plasticity through Fasciclin II-mediated cell adhesion. J Neurosci 2002; 22: 2496-2504.

47 Klagges BR, Heimbeck G, Godenschwege TA, Hofbauer A, Pflugfelder GO, Reifegerste $R$ et al. Invertebrate synapsins: a single gene codes for several isoforms in Drosophila. J Neurosci 1996; 16: 3154-3165.

48 O'Connor-Giles KM, Ho LL, Ganetzky B. Nervous wreck interacts with thickveins and the endocytic machinery to attenuate retrograde BMP signaling during synaptic growth. Neuron 2008; 58: 507-518.

49 Rodal AA, Motola-Barnes RN, Littleton JT. Nervous wreck and Cdc42 cooperate to regulate endocytic actin assembly during synaptic growth. J Neurosci 2008; 28: 8316-8325.

50 Winther ÅME, Vorontsova O, Rees KA, Näreoja T, Sopova E, Jiao W et al. An endocytic scaffolding protein together with Synapsin regulates synaptic vesicle clustering in the Drosophila neuromuscular junction. J Neurosci 2015; 35: 14756-14770.

$51 \mathrm{Kim}$ SJ, Atwoodb HL, Cooper RL. Assessing accurate sizes of synaptic vesicles in nerve terminals. Brain Res 2000; 877: 209-217.

52 Stanishneva-Konovalova TB, Kelley CF, Eskin TL, Messelaar EM, Wasserman SA, Sokolova OS et al. Coordinated autoinhibition of F-BAR domain membrane binding and WASp activation by Nervous Wreck. Proc Nat Acad Sci USA 2016; 113: E5552-E5561.

53 Gunnarsson C, Bruun CF. Molecular characterization and clinical features of a patient with an interstitial deletion of 3p25.3-p26.1. Am J Med Genet A 2010; 152A: 3110-3114.

54 Waltereit R, Kautt S, Bartsch D. Expression of MEGAP mRNA during embryonic development. Gene Expr Patterns 2008; 8: 307-310.

55 Carlson BR, Lloyd KE, Kruszewski A, Kim I-H, Rodriguiz RM, Heindel C et al. WRP/srGAP3 facilitates the initiation of spine development by an inverse F-BAR domain, and its loss impairs long-term memory. J Neurosci 2011; 31: 2447-2460.

56 Bacon C, Endris V, Andermatt I, Niederkofler V, Waltereit R, Bartsch D et al. Evidence for a role of srGAP3 in the positioning of commissural axons within the ventrolateral funiculus of the mouse spinal cord. PLOS ONE 2011; 6: e19887.

(c) (1) $(\Theta)$ This work is licensed under a Creative Commons Attribution-NonCommercial-NoDerivs 4.0 International License. The images or other third party material in this article are included in the article's Creative Commons license, unless indicated otherwise in the credit line; if the material is not included under the Creative Commons license, users will need to obtain permission from the license holder to reproduce the material. To view a copy of this license, visit http://creativecommons.org/licenses/by-nc-nd/4.0/

(C) The Author(s) 2018

Supplementary Information accompanies the paper on Experimental \& Molecular Medicine website (http://www.nature.com/emm) 